\title{
Highly diastereoselective 1,3-dipolar cycloaddition of nonstabilized azomethine ylides to 3-nitro-2-trihalomethyl-2H-chromenes: synthesis of 1-benzopyrano[3,4-c]pyrrolidines
}

\author{
Vladislav Yu. Korotaev ${ }^{\mathrm{a}}$, Alexey Yu. Barkov ${ }^{\mathrm{a}}$, Vladimir S. Moshkin ${ }^{\mathrm{a}}$, \\ Evgeniya G. Matochkina ${ }^{\mathrm{b}}$, Mikhail I. Kodess ${ }^{\mathrm{b}}$, Vyacheslav Ya. Sosnovskikh ${ }^{\mathrm{a}, *}$ \\ ${ }^{a}$ Department of Chemistry, Ural Federal University, 620000 Ekaterinburg, Russian Federation \\ ${ }^{\mathrm{b}}$ Institute of Organic Synthesis, Ural Branch of the Russian Academy of Sciences, 620041 Ekaterinburg, Russian Federation
}

\section{A R T I C L E I N F O}

\section{Article history:}

Received 17 May 2013

Received in revised form 8 July 2013

Accepted 22 July 2013

Available online 31 July 2013

\begin{abstract}
A B S T R A C T
Reactions of 3-nitro-2-trifluoro(trichloro)methyl-2H-chromenes, including 2-unsubstituted derivatives, with $N$-alkyl- $\alpha$-amino acids (sarcosine, proline) and paraformaldehyde proceed diastereoselectively to give 1-benzopyrano[3,4-c]pyrrolidines in good yields as a result of a 1,3-dipolar cycloaddition of the intermediate nonstabilized azomethine ylide at the $\Delta^{3}$-bond of the chromene system.
\end{abstract}

(c) 2013 Elsevier Ltd. All rights reserved.

Keywords:

3-Nitro-2H-chromenes

Nonstabilized azomethine ylides

1,3-Dipolar cycloaddition

1-Benzopyrano[3,4-c]pyrrolidines

\section{Introduction}

1,3-Dipolar cycloaddition is one of the most important methods for the synthesis of five-membered heterocycles. The high regioand stereoselectivity typical of these reactions make them an indispensable tool for synthesizing natural molecules with a few chiral centers. ${ }^{1}$ In particular, azomethine ylides derived from readily available $N$-alkyl- $\alpha$-amino acids and carbonyl compounds enable one-step syntheses of substituted pyrrolidines that are of considerable interest as compounds with a broad spectrum of bioactivity. $^{2}$ On the other hand, chromane derivatives are widespread in the plant world and also possess valuable biological and pharmacological properties. ${ }^{3}$ In view of this, combining benzopyran and pyrrolidine moieties in a single molecule seems a synthetic task of current interest. ${ }^{4}$ cis-Benzopyranopyrrolidine $\mathbf{I}$ is an antagonist of $5-\mathrm{HT}_{2 \mathrm{C}}$ receptors with respect to $5-\mathrm{HT}_{2 \mathrm{~A}}$, ${ }^{4 \mathrm{C}}$ whereas its more complex derivative II (Fiduxosin) is an $\alpha_{1}$ adrenoreceptor antagonist and shows an $\alpha_{1 \mathrm{a}} / \alpha_{1 \mathrm{~b}}$ selectivity for adrenoreceptors; it was suggested as a promising pharmaceutical agent for the treatment of benign prostatic hyperplasia ${ }^{5}$ (Fig. 1).

\footnotetext{
* Corresponding author. Fax: +7 34326159 78; e-mail addresses: vyacheslav. sosnovskikh@usu.ru, sosn1951@mail.ru (V.Ya. Sosnovskikh).
}<smiles>COc1cccc(OC)c1[C@H]1CN(Cc2ccccc2)C[C@H]1C</smiles><smiles>COc1cccc2c1[C@H]1CN(CCCCn3c(=O)[nH]c4c(sc5ncc(-c6ccccc6)nc54)c3=O)C[C@H]1CO2</smiles>

Fig. 1. Benzopyranopyrrolidine drugs candidates.

The key stage in the synthesis of benzopyranopyrrolidines is the cycloaddition of nonstabilized azomethine ylides to coumarins ${ }^{4,6}$ and $2 \mathrm{H}$-chromenes, however, information about such reactions in the $2 \mathrm{H}$-chromenes series is quite limited. It is only known that 2 aryl-3-nitro- $2 \mathrm{H}$-chromenes react with azomethine ylides at reflux in toluene for several hours to give the corresponding 4-aryl-3anitrobenzopyrano[3,4-c]pyrrolidines. ${ }^{7}$ Taking into account the literature data, ${ }^{8}$ we envisaged that introduction of such powerful electron-withdrawing substituents as $\mathrm{CF}_{3}$ and $\mathrm{CCl}_{3}$ groups into the 2-position of 3-nitro- $2 \mathrm{H}$-chromenes would increase their reactivity toward 1,3-dipolar cycloaddition with various 1,3-dipoles and open up a broad synthetic scope of these important oxygen-containing heterocycles. Of particular interest is the fact that introduction of a trifluoromethyl group into bioactive molecules can have profound and unexpected results on biological activity and reactivity of the 
derived fluorinated compounds. ${ }^{9}$ As a consequence, exploitation of an efficient method for the synthesis of these compounds is highly desirable.

\section{Results and discussion}

3-Nitro-2-trihalomethyl-2H-chromenes $\mathbf{1}(\mathrm{X}=\mathrm{F}, \mathrm{Cl})$, prepared by tandem condensation of the appropriate salicylaldehydes with 3,3,3-trifluoro(trichloro)-1-nitropropenes, ${ }^{10}$ have not received much attention despite their potential interest as $\mathrm{CF}_{3}$ - and $\mathrm{CCl}_{3}-$ building blocks in organic synthesis for the construction of trihalomethyl-containing heterocycles. The majority of the reactions with these compounds are nucleophilic additions at the 4position leading to various types of 4 -substituted chromanes. ${ }^{11}$ Most pertinent to the present research is the hetero-Diels-Alder reaction of 3-nitro-2-trihalomethyl-2H-chromenes $\mathbf{1}$ with electron-rich dienophiles, such as ethyl vinyl ether and 2,3dihydrofuran, providing a straightforward route to the cyclic nitronates. ${ }^{12}$ However, examples of the participation of chromenes $\mathbf{1}$ in any 1,3-dipolar cycloaddition reactions are lacking, a fact prompting us to investigate their reaction with the nonstabilized azomethine ylide derived from $N$-alkyl- $\alpha$-amino acids (sarcosine, proline) and paraformaldehyde, and elucidate the effects of different 3-nitro- $2 \mathrm{H}$-chromenes on the occurrence and stereoselectivity of cycloaddition reactions compared to those of 2-aryl-3-nitro- $2 \mathrm{H}$ chromenes. ${ }^{7}$

Herein, we have found that treatment of 3-nitro-2trihalomethyl-2H-chromenes $\mathbf{1 a}-\mathbf{h}$ with the azomethine ylide generated in situ from sarcosine and paraformaldehyde in refluxing toluene for 5-10 min with azeotropic removal of water affords the desired benzopyrano[3,4-c]pyrrolidines $\mathbf{2 a}-\mathbf{h}$ in high yields (77-97\%), which were isolated as free bases by crystallization from dichloromethane/hexane. This indicates that the presence of an electron-withdrawing trihalomethyl group at the 2-position increases the dipolarophilic reactivity of the $2-\mathrm{CX}_{3}$-chromenes $\mathbf{1 a}-\mathbf{h}$ toward the azomethine ylide, and makes these compounds more reactive than non-halogenated 3 -nitro- $2 \mathrm{H}$-chromenes. As is the case of cycloaddition reactions involving 2-aryl-3-nitro- $2 \mathrm{H}$-chromenes, ${ }^{7}$ compounds $\mathbf{2 a}-\mathbf{h}$ were formed mainly as the cis-isomers in relation to the nitro and trihalomethyl groups at C-3a and C-4. The synchronism of the reactions of nonstabilized azomethine ylides with alkenes results in the cis-fusion of the new pyrrolidine ring. The appearance of the trans-adducts $\mathbf{2}$ 'a $-\mathbf{h}$ (3-30\% according to the ${ }^{1} \mathrm{H}$ NMR data) was observed only in the crude products. Thus, the ready accessibility and high reactivity of $\mathrm{CX}_{3}$-chromenes $\mathbf{1 a}-\mathbf{h}$ have made them useful substrates for constructing biologically and medicinally important products (Scheme 1 ).

The structures of compounds $\mathbf{2} \mathbf{a}-\mathbf{h}$ were determined by elemental analysis (as their oxalates $\mathbf{3 a}-\mathbf{h}$, which were obtained in almost quantitative yield in acetone) and by comparison of their IR, ${ }^{1} \mathrm{H}$, and ${ }^{13} \mathrm{C}$ NMR spectra with data for related benzopyranopyrrolidines. ${ }^{6,7}$ Selected ${ }^{1} \mathrm{H}$ NMR spectroscopic data for compounds $\mathbf{2}$ and $\mathbf{2}^{\prime}$ as well as $\mathbf{3}$ and $\mathbf{3}^{\prime}$, which confirm the structure, are collected in Table 1 . The ${ }^{1} \mathrm{H}$ NMR spectra of cis-pyrrolidines $\mathbf{2}$ in $\mathrm{CDCl}_{3}$ contain the following characteristic signals: a triplet or a doublet of doublets of the $\mathrm{H}-1^{\prime}$ proton at $\delta 2.2-2.6 \mathrm{ppm}$ $(J=9.0-11.0 \mathrm{~Hz})$, which is subjected to the shielding effect of the benzene moiety in the cis-position; a triplet or a doublet of doublets of $\mathrm{H}-1^{\prime \prime}$ at $\delta 3.5-3.8 \mathrm{ppm}(J=7.7-9.0 \mathrm{~Hz})$; doublets of geminal protons $\mathrm{H}-3^{\prime}$ and $\mathrm{H}-3^{\prime \prime}$ at $\delta$ 2.7-3.0 and 4.0-4.5 ppm $(J=11.8-12.2 \mathrm{~Hz})$; a triplet or a doublet of doublets of the $\mathrm{H}-9 \mathrm{~b}$ benzyl proton at $\delta 4.0-4.2 \mathrm{ppm}(J=7.5-10.5 \mathrm{~Hz})$. In the case of trans-pyrrolidines $\mathbf{2}^{\prime}$, the strongly shielded proton $\mathrm{H}-1^{\prime}$ at $\delta 1.8-2.0 \mathrm{ppm}$ and strongly deshielded proton $\mathrm{H}-4$ at $\delta 4.8-5.2 \mathrm{ppm}$ (this atom exists in the cis-position toward the $\mathrm{NO}_{2}$ group) were observed as a consequence of the configuration change at C-4.

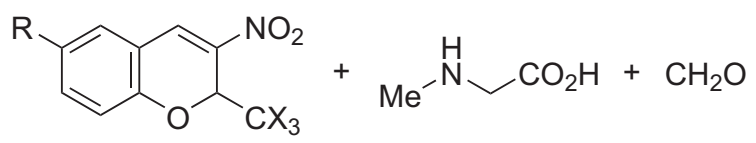

1a-h<smiles>C[14CH3]</smiles><smiles>[R]c1ccc2c(c1)[C@H]1CN(C)C[C@]1([N+](=O)[O-])[C@@H](C)O2</smiles><smiles>[R]c1ccc2c(c1)[C@H]1CN(C)C[C@]1([N+](=O)[O-])[C@@H](C)O2</smiles>

$$
\begin{aligned}
& \text { a: } X=F, R=H(97 \%) \\
& \text { b: } X=F, R=\operatorname{Br}(93 \%) \\
& \text { c: } X=F, R=M e O(77 \%) \\
& \text { d: } X=F, R=\mathrm{NO}_{2}(92 \%)
\end{aligned}
$$$$
\begin{aligned}
& \text { e: } X=C l, R=H(89 \%) \\
& \text { f : } X=C l, R=B r(80 \%) \\
& \text { g: } X=C l, R=M e O(85 \%) \\
& \text { h: } X=C l, R=\mathrm{NO}_{2}(78 \%)
\end{aligned}
$$

Scheme 1.

Significant variations are observed in the chemical shifts of $\mathrm{H}-4$ on going from bases cis-pyrrolidines $2\left(\delta 4.3-4.6 \mathrm{ppm}\right.$ in $\left.\mathrm{CDCl}_{3}\right)$ to their oxalates 3 ( $\delta 5.2-5.7 \mathrm{ppm}$ in DMSO- $d_{6}$ ). All signals in the ${ }^{1} \mathrm{H}$ and ${ }^{13} \mathrm{C}$ NMR spectra of compounds $\mathbf{2}$ and $\mathbf{3}$ were assigned on the basis of $2 \mathrm{D}^{1} \mathrm{H}-{ }^{13} \mathrm{C}$ HSQC and HMBC experiments for $\mathbf{2 b}, \mathbf{3 d}$, and $\mathbf{3 h}$. Moreover, the stereochemistry of $\mathbf{2 b}$ was unambiguously confirmed by X-ray single crystal analysis (Fig. 2).

In addition to sarcosine, proline was briefly examined in the reaction. Cycloaddition of the azomethine ylide derived from proline and paraformaldehyde with chromenes 1a,b,f and 3-nitro-2phenyl-2H-chromene (1i) occurred in a similar way to give compounds $\mathbf{4 a}-\mathbf{d}$, which were isolated from a mixture of regio- and stereoisomers in low yields (11-34\%). Obviously, in contrast to the azomethine ylide from sarcosine, this cycloaddition was less selective. Only the indicated diastereomer could be separated by recrystallization of the mixture from diethyl ether and hexane, however, product $\mathbf{4 c}$ was obtained as a 68:32 mixture of $\mathbf{4 c}$ with its minor isomer, the structure of which was not determined (Scheme 2).

The relative stereochemistry in cycloadducts $\mathbf{4 b}$ and $\mathbf{4 d}$ was established by $2 \mathrm{D}^{1} \mathrm{H}-{ }^{1} \mathrm{H}$ NOESY experiments. The most important proof of their stereochemistry was the NOE enhancements indicated with arrows in Fig. 3. All signals in the ${ }^{1} \mathrm{H}$ and ${ }^{13} \mathrm{C}$ NMR spectra of compounds 4 were assigned on the basis of $2 \mathrm{D}{ }^{1} \mathrm{H}-{ }^{1} \mathrm{H}$ COSY, ${ }^{1} \mathrm{H}-{ }^{13} \mathrm{C}$ HSQC, and HMBC experiments of $\mathbf{4 b} \mathbf{b}, \mathbf{d}$. The doublet of doublets or triplet of the $\mathrm{H}-11$ a benzyl proton in compounds $4 \mathbf{a}-\mathbf{d}$ allows us to rule out the alternative regioisomeric structure, in which the benzyl proton should interact with only one vicinal proton. It is necessary to be mentioned that in the case of chromenes 1a,e, sarcosine, and benzaldehyde the reaction led to a mixture of starting materials and unidentified products.

Next, taking into account the broad utility of benzopyranopyrrolidines in medicinal chemistry, ${ }^{4,5}$ and the fact that the literature contains no data on reactions of 2 -unsubstituted $2 \mathrm{H}$ chromenes with nonstabilized azomethine ylides, we studied the reaction of 3-cyano- and 3-methoxycarbonyl-2H-chromenes $\mathbf{1 j}, \mathbf{k}$ with sarcosine and paraformaldehyde. It was found that refluxing these compounds in benzene gave benzopyrano[3,4-c]pyrrolidines $\mathbf{5 a , b}$, which were isolated as hydrochlorides in high yields (76-83\%). The desired products precipitate upon neutralization of the reaction mixtures with $\mathrm{HCl}$, prepared in situ from $\mathrm{AcCl}$ and $i$ $\mathrm{PrOH}$, and a simple filtration provides analytically pure material. In 
Table 1

${ }^{1} \mathrm{H}$ NMR chemical shifts of protons $\mathrm{H}(1), \mathrm{H}(3), \mathrm{H}(9 \mathrm{~b})$, and $\mathrm{H}(4)$ in benzopyranopyrrolidines $\mathbf{2 a}-\mathbf{h}\left(\mathrm{CDCl}_{3}\right)$ and their oxalates $\mathbf{3 a}-\mathbf{h}\left(\mathrm{DMSO}^{-} \mathrm{d}_{6}\right)$

\begin{tabular}{|c|c|c|c|c|c|c|c|c|c|c|c|c|}
\hline Compd & \multicolumn{12}{|c|}{${ }^{1} \mathrm{H}$ NMR $(\delta, \mathrm{ppm})$} \\
\hline & \multicolumn{6}{|c|}{ Cis-isomer 2} & \multicolumn{6}{|c|}{ Trans-isomer $\mathbf{2}^{\prime}$} \\
\hline $2 a$ & 2.45 & 3.55 & 2.81 & 4.02 & 4.06 & 4.43 & 1.89 & 3.44 & 2.76 & $3.99-4.08$ & & 4.88 \\
\hline 2b & 2.48 & 3.50 & 2.85 & 3.97 & 4.02 & 4.43 & 1.92 & 3.43 & 2.74 & $3.94-4.05$ & & 4.85 \\
\hline $2 c$ & 2.43 & 3.54 & 2.79 & 4.02 & 4.05 & 4.39 & 1.89 & 3.44 & 2.74 & 4.01 & 3.96 & 4.82 \\
\hline 2d & 2.62 & 3.56 & 3.00 & 3.91 & 4.09 & 4.59 & 2.04 & 3.48 & 2.83 & 4.02 & 4.14 & 4.96 \\
\hline $2 e$ & 2.25 & 3.71 & 2.77 & 4.51 & 4.18 & 4.38 & 1.83 & 3.40 & 3.05 & 4.10 & 3.92 & 5.19 \\
\hline $2 f$ & 2.25 & 3.67 & 2.78 & 4.48 & 4.15 & 4.36 & 1.83 & 3.40 & 3.03 & 4.09 & 3.88 & 5.15 \\
\hline $3 \mathbf{b}$ & 2.45 & 3.73 & 2.88 & 4.14 & 4.05 & 5.46 & $\mathrm{a}$ & 3.44 & 2.72 & 3.92 & 4.24 & $\mathrm{a}$ \\
\hline $3 c$ & 2.44 & 3.75 & 2.88 & 4.14 & 3.99 & 5.32 & $\mathrm{a}$ & 3.46 & 2.67 & 3.92 & 4.15 & 5.28 \\
\hline 3d & 2.58 & 3.87 & 2.99 & 4.20 & 4.22 & 5.69 & $\mathrm{a}$ & 3.47 & 2.80 & 3.92 & 4.28 & $\mathrm{a}$ \\
\hline $3 \mathbf{e}$ & 2.46 & 3.78 & 3.15 & 4.40 & 4.02 & 5.33 & $\mathrm{a}$ & 3.41 & 3.00 & 4.00 & 4.09 & 5.22 \\
\hline $3 \mathbf{f}$ & 2.41 & 3.76 & 3.08 & 4.36 & 4.06 & 5.39 & $\mathrm{a}$ & 3.40 & 2.98 & 3.98 & 4.11 & 5.30 \\
\hline $3 g$ & 2.40 & 3.77 & 3.09 & 4.36 & 4.00 & 5.23 & $\mathrm{a}$ & 3.41 & 2.96 & $\mathrm{a}$ & a & 5.12 \\
\hline $3 \mathbf{h}$ & 2.52 & 3.89 & 3.17 & 4.40 & 4.19 & 5.63 & $\mathrm{a}$ & & 3.00 & 4.00 & $\mathrm{a}$ & 5.56 \\
\hline
\end{tabular}

a The signal is masked.

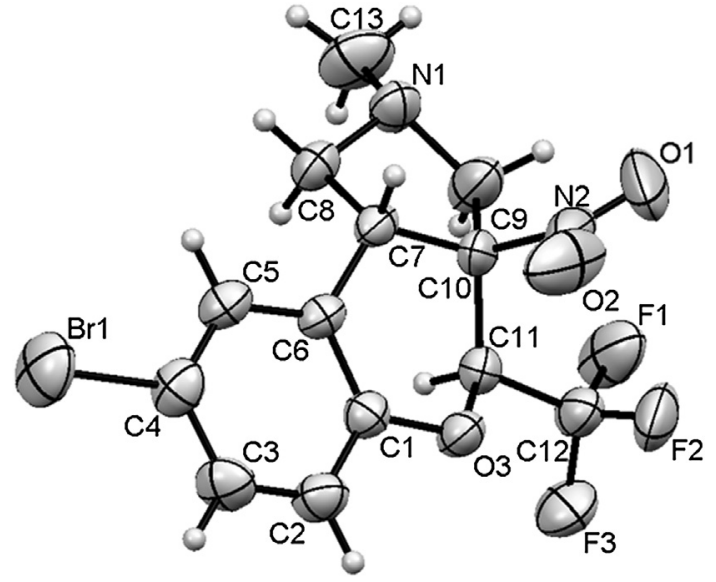

Fig. 2. X-ray crystal structure of cis-2b (ORTEP drawing, 50\% probability level).

contrast, the reaction of 3-formyl-2H-chromene (11) under the conditions described above afforded a 2.8:1 mixture of cycloadducts $\mathbf{5 c}$ and $\mathbf{6}$, respectively, which were not obtained as pure (Scheme 3).<smiles>[R]c1ccc2c(c1)C=C([N+](=O)[O-])C([R])O2</smiles>
$1 a, b, f, i$
4a: $\mathrm{R}=\mathrm{CF}_{3}, \mathrm{R}^{\prime}=\mathrm{H}(17 \%)$
4b: $R=\mathrm{CF}_{3}, \mathrm{R}^{\prime}=\mathrm{Br}(15 \%)$
4c: $\mathrm{R}=\mathrm{CCl}_{3}, \mathrm{R}^{\prime}=\mathrm{Br}(11 \%)$
4d: $R=P h, R^{\prime}=H(34 \%)$

Scheme 2.
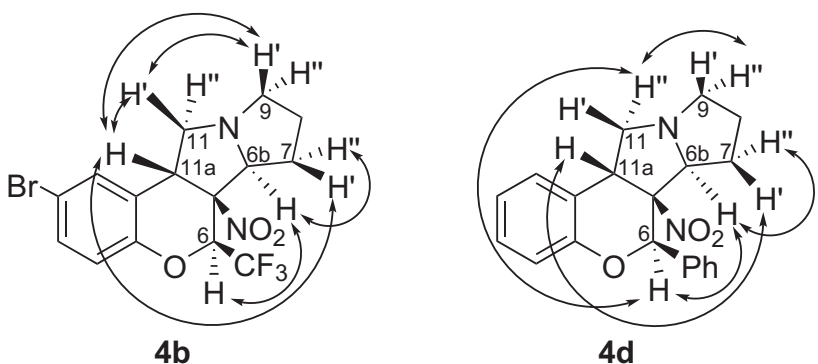

Fig. 3. NOE connectivities in compounds $\mathbf{4 b}$ and $\mathbf{4 d}$<smiles>[R]C1=Cc2ccccc2OC1</smiles>

\section{Conclusion}

In conclusion, the use of 3-nitro-2-trihalomethyl-2H-chromenes, including 2-unsubstituted derivatives, in 1,3-dipolar cycloadditions of nonstabilized azomethine ylides allows the 
construction of biologically interesting 1-benzopyrano[3,4-c]pyrrolidines in one pot three-component reaction.

\section{Experimental}

\subsection{General}

NMR spectra were recorded on Bruker DRX-400 $\left({ }^{1} \mathrm{H}-400 \mathrm{MHz}\right.$ $\left.{ }^{19} \mathrm{~F}-376 \mathrm{MHz}\right)$ and AVANCE-500 $\left({ }^{1} \mathrm{H}-500 \mathrm{MHz},{ }^{13} \mathrm{C}-126 \mathrm{MHz}\right)$ spectrometers in DMSO- $d_{6}$ and $\mathrm{CDCl}_{3}$ with TMS and $\mathrm{C}_{6} \mathrm{~F}_{6}$ as internal standards, respectively. IR spectra were recorded on a Perkin-Elmer Spectrum BX-II instrument as $\mathrm{KBr}$ discs. Elemental analysis was performed on Perkin-Elmer CHN PE 2400. All solvents used were dried and distilled per standard procedures. Melting points were determined without correction. The starting 3-nitrochromenes $\mathbf{1 a}-\mathbf{h}$ were prepared according to described procedures. ${ }^{10}$

\subsection{General procedure for the preparation of benzopyrano [3,4-c]pyrrolidines (2a-h)}

A mixture of sarcosine $(0.23 \mathrm{~g}, 2.6 \mathrm{mmol})$, paraformaldehyde $(0.19 \mathrm{~g}, 6.0 \mathrm{mmol})$, and the corresponding 3-nitrochromene $(1.0 \mathrm{mmol})$ was heated under reflux in toluene $(15 \mathrm{~mL})$ with a Dean-Stark trap for 5-10 min. After that the mixture was concentrated under reduced pressure and the solid formed was washed with dichloromethane/hexane (1:2) to give crude product as a mixture of two diastereomers $\mathbf{2}$ and $\mathbf{2}^{\prime}$. Cis-isomers $\mathbf{2} \mathbf{a}-\mathbf{h}$ were prepared after simple recrystallization from dichloromethane/ hexane $(1: 2)$ as a colorless powder. Elemental analyses for bases $\mathbf{2 a}-\mathbf{h}$ were performed for their oxalates $\mathbf{3 a}-\mathbf{h}$.

4.2.1. (3aS*,4S*,9bR*)-2-Methyl-3a-nitro-4-(trifluoromethyl)1,2,3,3a,4,9b-hexahydrochromeno[3,4-c]pyrrole (2a). Yield 97\%, mp 76-77 ${ }^{\circ} \mathrm{C}$; IR (KBr): $\nu=1585,1548,1494,1477,1457,1397,1374$, $1338 \mathrm{~cm}^{-1}$; ${ }^{1} \mathrm{H} \mathrm{NMR}\left(500 \mathrm{MHz}, \mathrm{CDCl}_{3}\right): \delta=2.38(\mathrm{~s}, 3 \mathrm{H}, \mathrm{Me}), 2.45(\mathrm{t}$, $\left.1 \mathrm{H}, \mathrm{H}-1^{\prime}, J=8.7 \mathrm{~Hz}\right), 2.81\left(\mathrm{~d}, 1 \mathrm{H}, \mathrm{H}-3^{\prime}, J=12.2 \mathrm{~Hz}\right), 3.55\left(\mathrm{t}, 1 \mathrm{H}, \mathrm{H}-1^{\prime \prime}\right.$, $J=8.7 \mathrm{~Hz}), 4.02\left(\mathrm{~d}, 1 \mathrm{H}, \mathrm{H}-3^{\prime \prime}, J=12.2 \mathrm{~Hz}\right), 4.05(\mathrm{t}, 1 \mathrm{H}, \mathrm{H}-9 \mathrm{~b}, J=8.7 \mathrm{~Hz})$, 4.43 (q, 1H, H-4, J=5.5 Hz), 7.05-7.12 (m, 2H, H-6, H-8), 7.15 (d, 1H, $\mathrm{H}-9, J=7.0 \mathrm{~Hz}), 7.24(\mathrm{t}, 1 \mathrm{H}, \mathrm{H}-7, J=7.7 \mathrm{~Hz}) ;{ }^{19} \mathrm{~F}$ NMR $(470.5 \mathrm{MHz}$, $\left.\mathrm{CDCl}_{3}\right): \delta=\mathbf{2 a}(90 \%) 89.3\left(\mathrm{~d}, \mathrm{CF}_{3}, J=5.5 \mathrm{~Hz}\right), \mathbf{2} \mathbf{a}(10 \%) 88.7\left(\mathrm{~d}, \mathrm{CF}_{3}\right.$, $J=6.3 \mathrm{~Hz}) ;{ }^{13} \mathrm{C}$ NMR $\left(126 \mathrm{MHz}, \mathrm{CDCl}_{3}\right): \delta=41.1(\mathrm{Me}), 44.0(\mathrm{C}-9 \mathrm{~b})$, $62.4\left(\mathrm{q}, \mathrm{C}-3,{ }^{4} J_{\mathrm{C}, \mathrm{F}}=2.3 \mathrm{~Hz}\right), 62.4(\mathrm{C}-1), 76.0\left(\mathrm{q}, \mathrm{C}-4,{ }^{2} \mathrm{~J}_{\mathrm{C}, \mathrm{F}}=32.4 \mathrm{~Hz}\right)$, 91.2 (C-3a), 117.5 (C-6), 122.0 (C-9a), 122.2 (q, CF,$\left.{ }^{1} J_{\mathrm{C}, \mathrm{F}}=282.9 \mathrm{~Hz}\right)$, 123.7 (C-8), 128.2 (C-9), 128.4 (C-7), 151.7 (C-5a).

4.2.2. (3aS*,4S*,9bR*)-8-Bromo-2-methyl-3a-nitro-4-(trifluoromethyl)-1,2,3,3a,4,9b-hexahydrochromeno[3,4-c]pyrrole (2b). Yield 93\%, mp 111-112 ${ }^{\circ} \mathrm{C}$; IR (KBr): $\nu=1552,1482,1463,1409,1388,1350$, $1333 \mathrm{~cm}^{-1}$; ${ }^{1} \mathrm{H}$ NMR $\left(500 \mathrm{MHz}, \mathrm{CDCl}_{3}\right): \delta=2.38(\mathrm{~s}, 3 \mathrm{H}, \mathrm{Me}), 2.48(\mathrm{t}$, $\left.1 \mathrm{H}, \mathrm{H}-1^{\prime}, J=8.7 \mathrm{~Hz}\right), 2.85\left(\mathrm{~d}, 1 \mathrm{H}, \mathrm{H}-3^{\prime}, J=11.8 \mathrm{~Hz}\right), 3.50\left(\mathrm{t}, 1 \mathrm{H}, \mathrm{H}-1^{\prime \prime}\right.$, $J=8.7 \mathrm{~Hz}$ ), 3.97 (d, 1H, H-3",$J=11.8 \mathrm{~Hz}), 4.02(\mathrm{t}, 1 \mathrm{H}, \mathrm{H}-9 \mathrm{~b}, J=8.1 \mathrm{~Hz})$, $4.43(\mathrm{q}, 1 \mathrm{H}, \mathrm{H}-4, J=5.7 \mathrm{~Hz}), 6.98(\mathrm{~d}, 1 \mathrm{H}, \mathrm{H}-6, J=8.8 \mathrm{~Hz}), 7.29(\mathrm{~d}, 1 \mathrm{H}, \mathrm{H}-$ 9, $J=1.9 \mathrm{~Hz}), 7.35$ (dd, $1 \mathrm{H}, \mathrm{H}-7, J=8.8,1.9 \mathrm{~Hz}) ;{ }^{19} \mathrm{~F}$ NMR $(376 \mathrm{MHz}$, $\left.\mathrm{CDCl}_{3}\right): \delta=\mathbf{2 b}(80 \%) 89.4\left(\mathrm{~d}, \mathrm{CF}_{3}, J=5.7 \mathrm{~Hz}\right), \mathbf{2} \mathbf{b}(20 \%) 88.7\left(\mathrm{~d}, \mathrm{CF}_{3}\right.$, $J=6.2 \mathrm{~Hz}$ ); ${ }^{13} \mathrm{C}$ NMR $\left(126 \mathrm{MHz}, \mathrm{CDCl}_{3}\right): \delta=41.0(\mathrm{Me}), 43.7$ (C-9b), $62.2(\mathrm{C}-1), 62.2$ (q, C-3, $\left.{ }^{4} J_{\mathrm{C}, \mathrm{F}}=2.4 \mathrm{~Hz}\right), 75.9\left(\mathrm{q}, \mathrm{C}-4,{ }^{2} \mathrm{~J}_{\mathrm{C}, \mathrm{F}}=32.5 \mathrm{~Hz}\right.$ ), 90.6 (C-3a), 116.1 (C-8), 119.3 (C-6), $122.0\left(\mathrm{q}, \mathrm{CF}_{3},{ }^{1} J_{\mathrm{C}, \mathrm{F}}=283.0 \mathrm{~Hz}\right)$, 124.2 (C-9a), 130.9 (C-9), 131.4 (C-7), 150.7 (C-5a).

4.2.3. (3aS*,4S*,9bR*)-2-Methyl-8-methoxy-3a-nitro-4-(trifluoromethyl)-1,2,3,3a,4,9b-hexahydrochromeno[3,4-c]pyrrole (2c). Yield 77\%, mp 87-88 ${ }^{\circ} \mathrm{C}$; IR (KBr): $\nu=1616,1549,1503,1481,1468,1425$, 1386, $1371 \mathrm{~cm}^{-1} ;{ }^{1} \mathrm{H}$ NMR (400 MHz, $\left.\mathrm{CDCl}_{3}\right): \delta=2.38(\mathrm{~s}, 3 \mathrm{H}, \mathrm{Me})$, 2.43 (t, 1H, H-1',$J=8.9 \mathrm{~Hz}), 2.79\left(\mathrm{~d}, 1 \mathrm{H}, \mathrm{H}-3^{\prime}, J=11.8 \mathrm{~Hz}\right), 3.54(\mathrm{t}, 1 \mathrm{H}$, $\left.\mathrm{H}-1^{\prime \prime}, J=8.6 \mathrm{~Hz}\right), 3.78(\mathrm{~s}, 3 \mathrm{H}, \mathrm{MeO}), 4.02\left(\mathrm{~d}, 1 \mathrm{H}, \mathrm{H}-3^{\prime \prime}, J=11.8 \mathrm{~Hz}\right), 4.05$ $(\mathrm{t}, 1 \mathrm{H}, \mathrm{H}-9 \mathrm{~b}, J=8.8 \mathrm{~Hz}), 4.39$ (q, 1H, H-4, J=5.7 Hz), $6.64(\mathrm{dd}, 1 \mathrm{H}, \mathrm{H}-9$,
$J=2.9,0.7 \mathrm{~Hz}$ ), 6.80 (ddd, $1 \mathrm{H}, \mathrm{H}-7, J=9.0,2.9,0.5 \mathrm{~Hz}), 7.02$ (d, 1H, H-6, $J=9.0 \mathrm{~Hz}) ;{ }^{19} \mathrm{~F}$ NMR $\left(376 \mathrm{MHz}, \mathrm{CDCl}_{3}\right): \delta=\mathbf{2 c}(91 \%) 89.3\left(\mathrm{~d}, \mathrm{CF}_{3}, J\right.$ $5.7 \mathrm{~Hz}), \mathbf{2}^{\prime} \mathbf{c}(9 \%) 88.7\left(\mathrm{~d}, \mathrm{CF}_{3}, J=6.4 \mathrm{~Hz}\right) ;{ }^{13} \mathrm{C} \mathrm{NMR}\left(126 \mathrm{MHz}, \mathrm{CDCl}_{3}\right)$ : $\delta=41.0,44.3,55.7,62.4,62.5\left(\mathrm{q}, \mathrm{C}-3,{ }^{4} \mathrm{~J}_{\mathrm{C}, \mathrm{F}}=2.2 \mathrm{~Hz}\right), 76.3(\mathrm{q}, \mathrm{C}-4$, $\left.{ }^{2} J_{C, F}=32.3 \mathrm{~Hz}\right), 91.2,112.7,114.2,118.3,122.2\left(\mathrm{q}, \mathrm{CF}_{3},{ }^{1} J_{\mathrm{C}, \mathrm{F}}=283.0 \mathrm{~Hz}\right)$, $122.8,145.7,155.7$.

4.2.4. (3aS*,4S*,9bR*)-2-Methyl-3a,8-dinitro-4-(trifluoromethyl)1,2,3,3a,4,9b-hexahydrochromeno[3,4-c]pyrrole (2d). Yield 92\%, mp 143-144 ${ }^{\circ} \mathrm{C}$; IR (KBr): $\nu=1586,1559,1522,1486,1464,1374$, $1343 \mathrm{~cm}^{-1} ;{ }^{1} \mathrm{H}$ NMR $\left(400 \mathrm{MHz}, \mathrm{CDCl}_{3}\right): \delta=2.41$ (s, 3H, Me), $2.62(\mathrm{dd}$, $1 \mathrm{H}, \mathrm{H}-1^{\prime}, J=9.1,7.7 \mathrm{~Hz}$ ), 3.00 (d, $1 \mathrm{H}, \mathrm{H}-3^{\prime}, J=11.8 \mathrm{~Hz}$ ), 3.56 (t, 1H, H-1", $J=8.8 \mathrm{~Hz}), 3.91\left(\mathrm{~d}, 1 \mathrm{H}, \mathrm{H}-3^{\prime \prime}, J=11.8 \mathrm{~Hz}\right), 4.05(\mathrm{t}, 1 \mathrm{H}, \mathrm{H}-9 \mathrm{~b}, J=7.9 \mathrm{~Hz})$, $4.59(\mathrm{q}, 1 \mathrm{H}, \mathrm{H}-4, J=5.6 \mathrm{~Hz}), 7.23(\mathrm{~d}, 1 \mathrm{H}, \mathrm{H}-6, J=9.0 \mathrm{~Hz}), 8.13(\mathrm{~d}, 1 \mathrm{H}, \mathrm{H}-$ 9, $J=2.6 \mathrm{~Hz}$ ), 8.16 (dd, $1 \mathrm{H}, \mathrm{H}-7, J=9.0,2.6 \mathrm{~Hz}) ;{ }^{19} \mathrm{~F}$ NMR $(376 \mathrm{MHz}$, $\left.\mathrm{CDCl}_{3}\right): \delta=\mathbf{2 d}(78 \%) 89.5\left(\mathrm{~d}, \mathrm{CF}_{3}, J=5.6 \mathrm{~Hz}\right), \mathbf{2}^{\prime} \mathbf{d}(27 \%) 88.5\left(\mathrm{~d}, \mathrm{CF}_{3}\right.$, $J=6.1 \mathrm{~Hz}$ ).

4.2.5. (3aS*,4S,9bR*)-2-Methyl-3a-nitro-4-(trichloromethyl)1,2,3,3a,4,9b-hexahydrochromeno[3,4-c]pyrrole (2e). Yield 89\%, mp 126-127 ${ }^{\circ} \mathrm{C}$; IR (KBr): $\nu=1585,1547,1491,1453,1333 \mathrm{~cm}^{-1} ;{ }^{1} \mathrm{H}$ NMR (400 MHz, $\mathrm{CDCl}_{3}$ ): $\delta=2.25$ (t, $1 \mathrm{H}, \mathrm{H}^{-1}{ }^{\prime}, J=9.6 \mathrm{~Hz}$ ), 2.42 (s, 3H, Me), $2.77\left(\mathrm{~d}, 1 \mathrm{H}, \mathrm{H}-3^{\prime}, J=12.0 \mathrm{~Hz}\right.$ ), 3.71 (ddd, $1 \mathrm{H}, \mathrm{H}-1^{\prime \prime}, J=8.7,7.7,1.0 \mathrm{~Hz}$ ), 4.18 (dd, $1 \mathrm{H}, \mathrm{H}-9 \mathrm{~b}, J=10.0,7.8 \mathrm{~Hz}$ ), 4.38 (s, 1H, H-4), 4.51 (dd, 1H, $\mathrm{H}^{\prime \prime}, \mathrm{J}=12.0,1.0 \mathrm{~Hz}$ ), 7.09-7.17 (m, 3H, H-6, H-8, H-9), 7.27 (m, 1H, $\mathrm{H}-7$ ).

4.2.6. ( $\left.3 a S^{*}, 4 S^{*}, 9 b R^{*}\right)$-8-Bromo-2-methyl-3a-nitro-4-(trichloromethyl)-1,2,3,3a,4,9b-hexahydrochromeno[3,4-c]pyrrole (2f). Yield $80 \%$ (2f/2'f $=90: 10), \operatorname{mp~} 191-192{ }^{\circ} \mathrm{C}$; IR (KBr): $\nu=1545,1480,1447,1408$, $1334 \mathrm{~cm}^{-1}$; ${ }^{1} \mathrm{H}$ NMR $\left(400 \mathrm{MHz}, \mathrm{CDCl}_{3}\right): \delta=2.25\left(\mathrm{t}, 1 \mathrm{H}, \mathrm{H}-1^{\prime}\right.$, $J=9.5 \mathrm{~Hz}), 2.42(\mathrm{~s}, 3 \mathrm{H}, \mathrm{Me}), 2.78\left(\mathrm{~d}, 1 \mathrm{H}, \mathrm{H}-3^{\prime}, J=12.0 \mathrm{~Hz}\right), 3.67(\mathrm{t}, 1 \mathrm{H}$, $\left.\mathrm{H}-1^{\prime \prime}, J=8.2 \mathrm{~Hz}\right), 4.15(\mathrm{t}, 1 \mathrm{H}, \mathrm{H}-9 \mathrm{~b}, J=8.8 \mathrm{~Hz}), 4.36(\mathrm{~s}, 1 \mathrm{H}, \mathrm{H}-4), 4.48(\mathrm{~d}$, $1 \mathrm{H}, \mathrm{H}-3^{\prime \prime}, J=12.0 \mathrm{~Hz}$ ), 7.04 (d, 1H, H-6, J=8.7 Hz), 7.27 (s, 1H, H-9), 7.37 (dd, $1 \mathrm{H}, \mathrm{H}-7, J=8.7,2.2 \mathrm{~Hz}) ;{ }^{13} \mathrm{C} \mathrm{NMR}\left(126 \mathrm{MHz}, \mathrm{CDCl}_{3}\right): \delta=41.1$, 46.7, 61.7, 65.2, 86.6, 94.3, 94.8, 116.3, 119.3, 124.6, 130.2, 131.4, 151.8.

4.2.7. ( $\left.3 a S^{*}, 4 S^{*}, 9 b R^{*}\right)$-2-Methyl-8-methoxy-3a-nitro-4-(trichloromethyl)-1,2,3,3a,4,9b-hexahydrochromeno[3,4-c]pyrrole (2g). Yield $85 \%\left(\mathbf{2 g} / \mathbf{2}^{\prime} \mathbf{g}=97: 3\right), \mathrm{mp} 157-158{ }^{\circ} \mathrm{C}$ (decomp.); IR (KBr): $\nu=1615$, 1544, 1499, 1469, 1422, $1336 \mathrm{~cm}^{-1}$; ${ }^{1} \mathrm{H}$ NMR (400 MHz, $\left.\mathrm{CDCl}_{3}\right)$ : $\delta=2.23\left(\mathrm{t}, 1 \mathrm{H}, \mathrm{H}-1^{\prime}, J=9.5 \mathrm{~Hz}\right), 2.42(\mathrm{~s}, 3 \mathrm{H}, \mathrm{Me}), 2.74\left(\mathrm{~d}, 1 \mathrm{H}, \mathrm{H}-3^{\prime}\right.$, $J=12.0 \mathrm{~Hz}$ ), 3.68 (ddd, $\left.1 \mathrm{H}, \mathrm{H}-1^{\prime \prime}, J=8.7,7.7,1.0 \mathrm{~Hz}\right), 3.79$ (s, 3H, MeO), 4.17 (dd, 1H, H-9b, J=9.7, $8.0 \mathrm{~Hz}$ ), 4.33 (s, 1H, H-4), 4.50 (dd, 1H, H$3^{\prime \prime}, J=12.0,1.0 \mathrm{~Hz}$ ), 6.63 (d, $1 \mathrm{H}, \mathrm{H}-9, J=2.8 \mathrm{~Hz}$ ), 6.81 (dd, $1 \mathrm{H}, \mathrm{H}-7$, $J=9.0,2.8 \mathrm{~Hz}), 7.08$ (d, $1 \mathrm{H}, \mathrm{H}-6, J=9.0 \mathrm{~Hz}) ;{ }^{13} \mathrm{C} \mathrm{NMR}(126 \mathrm{MHz}$, $\left.\mathrm{CDCl}_{3}\right): \delta=41.2,47.2,55.7,61.9,65.3,87.1,94.7,95.1,112.1,113.9$, $118.2,123.3,146.7,155.9$.

4.2.8. (3aS*,4S*,9bR*)-2-Methyl-3a,8-dinitro-4-(trichloromethyl)1,2,3,3a,4,9b-hexahydrochromeno[3,4-c]pyrrole (2h). Yield 78\% (2h/ $\mathbf{2}^{\prime} \mathbf{h}=70: 30$ ), $\mathrm{mp}$ 217-218 ${ }^{\circ} \mathrm{C}$ (decomp.); IR (KBr): $\nu=1587,1546$, $1517,1485,1341 \mathrm{~cm}^{-1} ;{ }^{1} \mathrm{H}$ NMR $\left(400 \mathrm{MHz}, \mathrm{CDCl}_{3}\right): \delta=2.34(\mathrm{t}, 1 \mathrm{H}, \mathrm{H}-$ $1^{\prime}, J=9.5 \mathrm{~Hz}$ ), 2.45 (s, 3H, Me), 2.90 (d, 1H, H-3', J=12.1 Hz), 3.76 (t, $\left.1 \mathrm{H}, \mathrm{H}-1^{\prime \prime}, J=8.4 \mathrm{~Hz}\right), 4.21$ (t, $\left.1 \mathrm{H}, \mathrm{H}-9 \mathrm{~b}, J=8.9 \mathrm{~Hz}\right), 4.21$ (d, $1 \mathrm{H}, \mathrm{H}-3^{\prime \prime}$, $J=12.1 \mathrm{~Hz}), 4.48(\mathrm{~s}, 1 \mathrm{H}, \mathrm{H}-4), 7.29$ (d, $1 \mathrm{H}, \mathrm{H}-6, J=9.0 \mathrm{~Hz}), 8.11(\mathrm{~d}, 1 \mathrm{H}$, $\mathrm{H}-9, J=2.6 \mathrm{~Hz}$ ), 8.19 (dd, 1H, H-7, J=9.0, 2.6 Hz).

\subsection{General procedure for the preparation of benzopyrano [3,4-c]pyrrolidines oxalates (3a-h)}

Anhydrous oxalic acid $(0.10 \mathrm{~g}, 1.1 \mathrm{mmol})$ dissolved in hot acetone $(1.0 \mathrm{~mL})$ was added with stirring to the solution of the corresponding benzopyrano[3,4-c]pyrrolidine $2(1.0 \mathrm{mmol})$ in acetone $(0.5 \mathrm{~mL})$ and the solid formed was filtered off and washed with dry acetone to give compounds $\mathbf{3}$ as a white powder. 
4.3.1. (3aS*,4S*,9bR*)-2-Methyl-3a-nitro-4-(trifluoromethyl)1,2,3,3a,4,9b-hexahydrochromeno[3,4-c]pyrrole oxalate (3a). Yield 92\%, mp 197-198 ${ }^{\circ} \mathrm{C}$ (decomp.); IR (KBr): $\nu=1713,1641,1591,1564$, 1558, 1496, 1459, $1345 \mathrm{~cm}^{-1} ;{ }^{1} \mathrm{H}$ NMR (400 MHz, DMSO-d $\left.d_{6}\right): \delta=2.38$ (s, 3H, Me), $2.45\left(\mathrm{t}, 1 \mathrm{H}, \mathrm{H}-1^{\prime}, J=9.7 \mathrm{~Hz}\right), 2.88\left(\mathrm{~d}, 1 \mathrm{H}, \mathrm{H}-3^{\prime}, J=12.2 \mathrm{~Hz}\right)$, $3.73\left(\mathrm{t}, 1 \mathrm{H}, \mathrm{H}-1^{\prime \prime}, J=8.2 \mathrm{~Hz}\right.$ ), 4.02 (dd, $1 \mathrm{H}, \mathrm{H}-9 \mathrm{~b}, J=9.7,8.2 \mathrm{~Hz}$ ), 4.15 (d, $1 \mathrm{H}, \mathrm{H}-3^{\prime \prime}, J=12.2 \mathrm{~Hz}$ ), 5.42 (q, $1 \mathrm{H}, \mathrm{H}-4, J=6.0 \mathrm{~Hz}$ ), 7.12 (d, 1H, H-6, $J=8.2 \mathrm{~Hz}$ ), 7.14 (t, 1H, H-8, J=7.3 Hz), 7.28 (t, 1H, H-7, J=7.6 Hz), 7.34 (d, $1 \mathrm{H}, \mathrm{H}-9, J=7.6 \mathrm{~Hz}) ;{ }^{19} \mathrm{~F}$ NMR (376 MHz, DMSO- $\left.d_{6}\right): \delta=\mathbf{3 a}(96 \%)$ 90.1 (d, $\mathrm{CF}_{3}, J=6.0 \mathrm{~Hz}$ ), 3'a (4\%) 90.4 (d, $\left.\mathrm{CF}_{3}, J=6.7 \mathrm{~Hz}\right)$. Found: $\mathrm{C}$, 45.89; $\mathrm{H}, 3.74 ; \mathrm{N}, 7.05$. Calcd for $\mathrm{C}_{13} \mathrm{H}_{13} \mathrm{~F}_{3} \mathrm{~N}_{2} \mathrm{O}_{3} \cdot\left(\mathrm{CO}_{2} \mathrm{H}\right)_{2}: \mathrm{C}, 45.93 ; \mathrm{H}$, $3.85 ; \mathrm{N}, 3.14$

4.3.2. (3aS*,4S*,9bR*)-8-Bromo-2-methyl-3a-nitro-4-(trifluoromethyl)-1,2,3,3a,4,9b-hexahydrochromeno[3,4-c]pyrrole oxalate (3b). Yield 92\%, mp 187-188 ${ }^{\circ} \mathrm{C}$ (decomp.); IR ( $\left.\mathrm{KBr}\right): \nu=1713,1641,1566$, $1488,1345 \mathrm{~cm}^{-1} ;{ }^{1} \mathrm{H}$ NMR (400 MHz, DMSO- $\left.d_{6}\right): \delta=2.38$ (s, 3H, Me), $2.45\left(\mathrm{t}, 1 \mathrm{H}, \mathrm{H}-1^{\prime}, J=9.7 \mathrm{~Hz}\right), 2.88\left(\mathrm{~d}, 1 \mathrm{H}, \mathrm{H}-3^{\prime}, J=12.2 \mathrm{~Hz}\right), 3.73(\mathrm{t}, 1 \mathrm{H}$, $\mathrm{H}-1^{\prime \prime}, J=8.2 \mathrm{~Hz}$ ), 4.05 (dd, 1H, H-9b, J=9.0, 8.6 Hz), 4.14 (d, 1H, H-3" , $J=12.2 \mathrm{~Hz}$ ), 5.46 (q, 1H, H-4, J=6.0 Hz), 7.11 (d, 1H, H-6, $J=8.7 \mathrm{~Hz}$ ), 7.46 (dd, $1 \mathrm{H}, \mathrm{H}-7, J=8.7,2.2 \mathrm{~Hz}$ ), 7.60 (d, $1 \mathrm{H}, \mathrm{H}-9, J=2.2 \mathrm{~Hz}$ ); (4\%) 2.30 (s, 3H, Me), 2.72 (d, 1H, H-3', J=12.2 Hz), 3.44 (t, 1H, H-1" ${ }^{\prime \prime}, J=8.8 \mathrm{~Hz}$ ), $3.92\left(\mathrm{~d}, 1 \mathrm{H}, \mathrm{H}-3^{\prime \prime}, J=12.2 \mathrm{~Hz}\right), 4.24(\mathrm{t}, 1 \mathrm{H}, \mathrm{H}-9 \mathrm{~b}, J=8.2 \mathrm{~Hz}), 7.62$ (d, $1 \mathrm{H}, \mathrm{H}-9, J=2.2 \mathrm{~Hz}$ ); ${ }^{19} \mathrm{~F}$ NMR (376 MHz, DMSO- $\left.d_{6}\right): \delta=\mathbf{3 b}(96 \%) 90.1$ (d, $\left.\mathrm{CF}_{3}, J=6.0 \mathrm{~Hz}\right), \mathbf{3}^{\prime} \mathbf{b}$ (4\%) $90.4\left(\mathrm{~d}, \mathrm{CF}_{3}, J=6.7 \mathrm{~Hz}\right.$ ). Found: C, 38.42; $\mathrm{H}, 2.88 ; \mathrm{N}, 5.86$. Calcd for $\mathrm{C}_{13} \mathrm{H}_{12} \mathrm{BrF}_{3} \mathrm{~N}_{2} \mathrm{O}_{3} \cdot\left(\mathrm{CO}_{2} \mathrm{H}\right)_{2}: \mathrm{C}, 38.24 ; \mathrm{H}$, $2.99 ; \mathrm{N}, 5.95$.

4.3.3. ( $\left.3 a S^{*}, 4 S^{*}, 9 b R^{*}\right)$-2-Methyl-8-methoxy-3a-nitro-4-(trifluoromethyl)-1,2,3,3a,4,9b-hexahydrochromeno[3,4-c]pyrrole oxalate (3c). Yield 98\%, mp 171-172 ${ }^{\circ} \mathrm{C}$ (decomp.); IR (KBr): $\nu=1727,1617,1567$, 1504, 1461, 1438, $1345 \mathrm{~cm}^{-1}$; ${ }^{1} \mathrm{H}$ NMR (400 MHz, DMSO-d $): \delta=2.38$ (s, 3H, Me), $2.44\left(\mathrm{t}, 1 \mathrm{H}, \mathrm{H}-1^{\prime}, J=9.6 \mathrm{~Hz}\right), 2.88\left(\mathrm{~d}, 1 \mathrm{H}, \mathrm{H}-3^{\prime}, J=12.0 \mathrm{~Hz}\right)$, 3.73 (s, 3H, MeO), 3.75 (t, $1 \mathrm{H}, \mathrm{H}-1^{\prime \prime}, J=8.5 \mathrm{~Hz}$ ), 3.99 (dd, $1 \mathrm{H}, \mathrm{H}-9 \mathrm{~b}$, $J=9.4,8.4 \mathrm{~Hz}$ ), 4.14 (d, 1H, H-3", $J=12.0 \mathrm{~Hz}), 5.32$ (q, 1H, H-4, $J=6.0 \mathrm{~Hz}$ ), 6.85 (dd, 1H, H-7, J=8.9, $2.8 \mathrm{~Hz}), 6.90(\mathrm{~d}, 1 \mathrm{H}, \mathrm{H}-9$, $J=2.8 \mathrm{~Hz}$ ), 7.04 (d, 1H, H-6, $J=8.9 \mathrm{~Hz}) ;{ }^{19} \mathrm{~F}$ NMR (376 MHz, DMSO$\left.d_{6}\right): \delta=3 c(96 \%) 90.1$ (d, $\left.\mathrm{CF}_{3}, J=6.0 \mathrm{~Hz}\right), \mathbf{3}^{\prime} \mathbf{c}(4 \%) 90.4$ (d, $\mathrm{CF}_{3}$, $J=6.6 \mathrm{~Hz}) ;{ }^{13} \mathrm{C}$ NMR $\left(126 \mathrm{MHz}\right.$, DMSO- $\left.d_{6}\right): \delta=40.3,44.4,55.5,60.3$, 60.6, 74.6 (q, C-4, $\left.{ }^{2} J_{C, F}=31.4 \mathrm{~Hz}\right), 91.1,112.8,114.3,117.6,122.1,122.4$ $\left(\mathrm{q}, \mathrm{CF}_{3},{ }^{1} J_{\mathrm{C}, \mathrm{F}}=282.2 \mathrm{~Hz}\right), 145.3,155.1,161.5(\mathrm{C}=\mathrm{O})$. Found: $\mathrm{C}, 45.26$; $\mathrm{H}, 3.83$; N, 6.55. Calcd for $\mathrm{C}_{14} \mathrm{H}_{15} \mathrm{~F}_{3} \mathrm{~N}_{2} \mathrm{O}_{4} \cdot\left(\mathrm{CO}_{2} \mathrm{H}\right)_{2}$ : C, 45.51; $\mathrm{H}, 4.06$; $\mathrm{N}, 6.63$.

4.3.4. ( $\left.3 a S^{*}, 4 S^{*}, 9 b R^{*}\right)-2-M e t h y l-3 a, 8$-dinitro-4-(trifluoromethyl)1,2,3,3a,4,9b-hexahydrochromeno[3,4-c]pyrrole oxalate (3d). Yield 93\%, mp 158-159 ${ }^{\circ} \mathrm{C}$; IR (KBr): $\nu=1707,1639,1593,1574,1533,1493$, $1344 \mathrm{~cm}^{-1}$; ${ }^{1} \mathrm{H}$ NMR (500 MHz, DMSO- $\left.d_{6}\right): \delta=2.42$ (s, 3H, Me), 2.58 $\left(\mathrm{t}, 1 \mathrm{H}, \mathrm{H}-1^{\prime}, J=9.7 \mathrm{~Hz}\right), 2.99\left(\mathrm{~d}, 1 \mathrm{H}, \mathrm{H}-3^{\prime}, J=12.1 \mathrm{~Hz}\right), 3.87\left(\mathrm{t}, 1 \mathrm{H}, \mathrm{H}-1^{\prime \prime}\right.$, $J=8.1 \mathrm{~Hz}), 4.20\left(\mathrm{~d}, 1 \mathrm{H}, \mathrm{H}-3^{\prime \prime}, J=12.1 \mathrm{~Hz}\right), 4.22(\mathrm{t}, 1 \mathrm{H}, \mathrm{H}-9 \mathrm{~b}, J=9.8 \mathrm{~Hz})$, $5.69(\mathrm{q}, 1 \mathrm{H}, \mathrm{H}-4, J=5.9 \mathrm{~Hz}), 7.41(\mathrm{~d}, 1 \mathrm{H}, \mathrm{H}-6, J=8.9 \mathrm{~Hz}), 8.18(\mathrm{dd}, 1 \mathrm{H}$, $\mathrm{H}-7, J=8.9,2.1 \mathrm{~Hz}$ ), 8.36 (d, 1H, H-9, J=2.1 Hz), 12.45 (br s, 2H, OH); ${ }^{19} \mathrm{~F}$ NMR (376 MHz, DMSO- $\left.d_{6}\right): \delta=\mathbf{3 d}(98 \%) 90.2\left(\mathrm{~d}, \mathrm{CF}_{3}, J=5.9 \mathrm{~Hz}\right)$, $\mathbf{3}^{\prime} \mathbf{d}(2 \%) 90.4\left(\mathrm{~d}, \mathrm{CF}_{3}, J=6.6 \mathrm{~Hz}\right) ;{ }^{13} \mathrm{C}$ NMR (126 MHz, DMSO- $\left.d_{6}\right)$ : $\delta=40.3(\mathrm{Me}), 44.0(\mathrm{C}-9 \mathrm{~b}), 60.2\left(\mathrm{q}, \mathrm{C}-3,{ }^{4} \mathrm{~J}_{\mathrm{C}, \mathrm{F}}=2.3 \mathrm{~Hz}\right), 60.4(\mathrm{C}-1), 74.6$ (q, C-4, $\left.{ }^{2} J_{C, F}=31.7 \mathrm{~Hz}\right), 90.0$ (C-3a), $118.13(\mathrm{C}-6), 122.2\left(\mathrm{q}, \mathrm{CF}_{3}\right.$, ${ }^{1} J_{C, F}=282.1 \mathrm{~Hz}$ ), 122.5 (C-9a), 124.0 (C-7), 125.0 (C-9), 142.9 (C-8), 156.1 (C-5a), 161.4 ( $C=0$ ). Found: C, 41.12; H, 3.05; N, 9.29. Calcd for $\mathrm{C}_{13} \mathrm{H}_{12} \mathrm{~F}_{3} \mathrm{~N}_{3} \mathrm{O}_{5} \cdot\left(\mathrm{CO}_{2} \mathrm{H}\right)_{2}: \mathrm{C}, 41.20 ; \mathrm{H}, 3.23 ; \mathrm{N}, 9.61$.

4.3.5. (3aS*,4S*,9bR*)-2-Methyl-3a-nitro-4-(trichloromethyl)1,2,3,3a,4,9b-hexahydrochromeno[3,4-c]pyrrole oxalate (3e). Yield 96\%, mp 143-144 ${ }^{\circ} \mathrm{C}$ (decomp.); IR (KBr): $\nu=1719,1635,1591,1561$, $1496,1459,1366,1331 \mathrm{~cm}^{-1} ;{ }^{1} \mathrm{H}$ NMR (400 MHz, DMSO-d $): \delta=2.41$ (s, 3H, Me), 2.46 (dd, $\left.1 \mathrm{H}, \mathrm{H}-1^{\prime}, J=10.5,9.0 \mathrm{~Hz}\right), 3.15$ (d, $1 \mathrm{H}, \mathrm{H}-3^{\prime}$, $J=12.2 \mathrm{~Hz}$ ), 3.78 (t, $1 \mathrm{H}, \mathrm{H}-1^{\prime \prime}, J=8.0 \mathrm{~Hz}$ ), 4.05 (dd, $1 \mathrm{H}, \mathrm{H}-9 \mathrm{~b}, J=10.5$,
$7.4 \mathrm{~Hz}$ ), 4.40 (d, 1H, H-3" $\left.{ }^{\prime \prime} J=12.2 \mathrm{~Hz}\right), 5.33$ (s, 1H, H-4), 7.11 (d, 1H, H$6, J=8.2 \mathrm{~Hz}$ ), $7.14(\mathrm{t}, 1 \mathrm{H}, \mathrm{H}-8, J=7.6 \mathrm{~Hz}), 7.27-7.33(\mathrm{~m}, 2 \mathrm{H}, \mathrm{H}-7, \mathrm{H}-9)$. Found: $\mathrm{C}, 40.62 ; \mathrm{H}, 3.34 ; \mathrm{N}, 6.17$. Calcd for $\mathrm{C}_{13} \mathrm{H}_{13} \mathrm{Cl}_{3} \mathrm{~N}_{2} \mathrm{O}_{3} \cdot\left(\mathrm{CO}_{2} \mathrm{H}\right)_{2}$ : C, 40.79; H, 3.42; N, 6.34.

4.3.6. (3aS*,4S*,9bR*)-8-Bromo-2-methyl-3a-nitro-4-(trichloromethyl)-1,2,3,3a,4,9b-hexahydrochromeno[3,4-c]pyrrole oxalate (3f). Yield 95\%, mp $175-176{ }^{\circ} \mathrm{C}$ (decomp.); IR (KBr): $\nu=1711,1635,1563$, 1484, 1363, $1340 \mathrm{~cm}^{-1} ;{ }^{1} \mathrm{H}$ NMR (400 MHz, DMSO- $\left.d_{6}\right): \delta=2.38$ (s, $3 \mathrm{H}, \mathrm{Me}), 2.41\left(\mathrm{t}, 1 \mathrm{H}, \mathrm{H}-1^{\prime}, J=9.9 \mathrm{~Hz}\right), 3.08\left(\mathrm{~d}, 1 \mathrm{H}, \mathrm{H}-3^{\prime}, J=12.2 \mathrm{~Hz}\right)$, 3.76 (t, 1H, H-1" ${ }^{\prime \prime} J=7.9 \mathrm{~Hz}$ ), 4.06 (dd, 1H, H-9b, J=9.9, 8.3 Hz), 4.36 $\left(\mathrm{d}, 1 \mathrm{H}, \mathrm{H}-3^{\prime \prime}, J=12.2 \mathrm{~Hz}\right), 5.39$ (s, 1H, H-4), 7.10 (d, 1H, H-6, J=8.7 Hz), 7.46 (dd, $1 \mathrm{H}, \mathrm{H}-7, J=8.7,1.2 \mathrm{~Hz}$ ), 7.54 (br s, $1 \mathrm{H}, \mathrm{H}-9$ ); ${ }^{13} \mathrm{C}$ NMR $\left(126 \mathrm{MHz}\right.$, DMSO- $\left.d_{6}\right): \delta=40.4,46.4,59.8,63.1,84.1,93.9,95.0,115.1$, 119.0, 124.8, 130.5, 130.8, 151.4, $161.4(\mathrm{C}=\mathrm{O})$. Found: C, 34.95; $\mathrm{H}$, 2.72; N, 5.38. Calcd for $\mathrm{C}_{13} \mathrm{H}_{12} \mathrm{BrCl}_{3} \mathrm{~N}_{2} \mathrm{O}_{3} \cdot\left(\mathrm{CO}_{2} \mathrm{H}\right)_{2}$ : C, 34.61; $\mathrm{H} 2.71$; $\mathrm{N}, 5.38$.

4.3.7. (3aS*,4S*,9bR*)-2-Methyl-8-methoxy-3a-nitro-4-(trichloromethyl)-1,2,3,3a,4,9b-hexahydrochromeno[3,4-clpyrrole oxalate (3g). Yield 96\%, mp 172-173 ${ }^{\circ} \mathrm{C}$ (decomp.); IR (KBr): $\nu=1726,1617,1563$, $1503,1459,1434,1335 \mathrm{~cm}^{-1} ;{ }^{1} \mathrm{H}$ NMR $\left(400 \mathrm{MHz}\right.$, DMSO- $\left.d_{6}\right): \delta=2.38$ (s, 3H, Me), 2.40 (t, $\left.1 \mathrm{H}, \mathrm{H}-1^{\prime}, J=10.8 \mathrm{~Hz}\right), 3.09\left(\mathrm{~d}, 1 \mathrm{H}, \mathrm{H}-3^{\prime}, J=12.2 \mathrm{~Hz}\right)$, 3.74 (s, 3H, MeO), 3.77 (t, 1H, H-1",$J=8.4 \mathrm{~Hz}$ ), 4.00 (dd, 1H, H-9b, $J=10.0,7.8 \mathrm{~Hz}$ ), 4.36 (d, $\left.1 \mathrm{H}, \mathrm{H}-3^{\prime \prime}, J=12.2 \mathrm{~Hz}\right), 5.23$ (s, 1H, H-4), 6.84-6.88 (m, 2H, H-7, H-9), 7.04 (d, 1H, H-6, J=8.8 Hz); ${ }^{13} \mathrm{C}$ NMR $\left(126 \mathrm{MHz}\right.$, DMSO- $\left.d_{6}\right): \delta=40.3,46.8,55.6,59.9,62.9,84.6,94.0,95.3$ 112.3, 114.1, 117.5, 122.8, 146.1, 155.2, $161.7(\mathrm{C}=\mathrm{O})$. Found: $\mathrm{C}$, 40.46; $\mathrm{H}, 3.42 ; \mathrm{N}, 5.87$. Calcd for $\mathrm{C}_{14} \mathrm{H}_{15} \mathrm{Cl}_{3} \mathrm{~N}_{2} \mathrm{O}_{4} \cdot\left(\mathrm{CO}_{2} \mathrm{H}\right)_{2}: \mathrm{C}, 40.74 ; \mathrm{H}, 3.63$; N, 5.94 .

4.3.8. (3aS*,4S*,9bR*)-2-Methyl-3a,8-dinitro-4-(trichloromethyl)1,2,3,3a,4,9b-hexahydrochromeno[3,4-clpyrrole oxalate (3h). Yield 92\%, mp 165-166 ${ }^{\circ} \mathrm{C}$ (decomp.); IR ( $\left.\mathrm{KBr}\right): \nu=1715,1632,1590,1563$, 1541, 1488, $1345 \mathrm{~cm}^{-1}$; ${ }^{1} \mathrm{H}$ NMR (500 MHz, DMSO-d $): \delta=2.42(\mathrm{~s}$, $3 \mathrm{H}, \mathrm{Me}), 2.52\left(\mathrm{t}, 1 \mathrm{H}, \mathrm{H}-1^{\prime}, J=9.3 \mathrm{~Hz}\right), 3.17\left(\mathrm{~d}, 1 \mathrm{H}, \mathrm{H}^{\prime} 3^{\prime}, J=12.2 \mathrm{~Hz}\right)$, 3.89 (t, 1H, H-1",$J=8.0 \mathrm{~Hz}$ ), 4.19 (dd, $1 \mathrm{H}, \mathrm{H}-9 \mathrm{~b}, J=10.2,8.0 \mathrm{~Hz}), 4.40$ $\left(\mathrm{d}, 1 \mathrm{H}, \mathrm{H}-3^{\prime \prime}, J=12.2 \mathrm{~Hz}\right), 5.63(\mathrm{~s}, 1 \mathrm{H}, \mathrm{H}-4), 7.39(\mathrm{~d}, 1 \mathrm{H}, \mathrm{H}-6, J=9.0 \mathrm{~Hz})$, 8.19 (dd, $1 \mathrm{H}, \mathrm{H}-7, J=9.0,2.1 \mathrm{~Hz}), 8.28(\mathrm{~d}, 1 \mathrm{H}, \mathrm{H}-9, J=2.1 \mathrm{~Hz}) ;{ }^{13} \mathrm{C} \mathrm{NMR}$ $\left(126 \mathrm{MHz}\right.$, DMSO- $\left.d_{6}\right): \delta=40.4(\mathrm{Me}), 46.5(\mathrm{C}-9 \mathrm{~b}), 59.8(\mathrm{C}-1), 63.2(\mathrm{C}-$ 3), 83.9 (C-4), 93.3 (C-3a), $94.7\left(\mathrm{CCl}_{3}\right), 118.0$ (C-6), 123.3 (C-9a), 124.0 (C-7), 124.3 (C-9), 142.9 (C-8), 156.9 (C-5a), 161.5(C=0). Found: C, 37.23; $\mathrm{H}, 2.73 ; \mathrm{N}, 8.49$. Calcd for $\mathrm{C}_{13} \mathrm{H}_{12} \mathrm{Cl}_{3} \mathrm{~N}_{3} \mathrm{O}_{5} \cdot\left(\mathrm{CO}_{2} \mathrm{H}\right)_{2}$ : C, 37.02; $\mathrm{H}, 2.90 ; \mathrm{N}, 8.63$.

\subsection{Compounds $4 a-d$}

4.4.1. (6S*,6aS*,6bS*,11aR*)-6a-Nitro-6-(trifluoromethyl)-6,6a,6b,7,8,9,11,11a-octahydrochromeno[3,4-a]pyrrolizine (4a). This compound was prepared from 3-nitro-2-(trifluoromethyl)-2H-chromene (1a), proline, and paraformaldehyde according to the described procedure for compounds 2 . Yield $17 \%, \mathrm{mp} 136-137{ }^{\circ} \mathrm{C}$, colorless needles; IR (KBr): $\nu=1587,1549,1490,1458,1376 \mathrm{~cm}^{-1} ;{ }^{1} \mathrm{H}$ $\operatorname{NMR}\left(400 \mathrm{MHz}, \mathrm{CDCl}_{3}\right): \delta=1.49-3.12\left(\mathrm{~m}, 6 \mathrm{H}, 3 \mathrm{CH}_{2}\right), 3.30(\mathrm{dd}, 1 \mathrm{H}, \mathrm{H}-$ $11^{\prime}, J=10.7,5.0 \mathrm{~Hz}$ ), 3.59 (dd, $\left.1 \mathrm{H}, \mathrm{H}-11^{\prime \prime}, J=10.7,7.3 \mathrm{~Hz}\right), 4.20$ (t, $1 \mathrm{H}, \mathrm{H}-$ $6 \mathrm{~b}, J=7.4 \mathrm{~Hz}), 4.27(\mathrm{t}, 1 \mathrm{H}, \mathrm{H}-11 \mathrm{a}, J=6.1 \mathrm{~Hz}), 4.88(\mathrm{q}, 1 \mathrm{H}, \mathrm{H}-6$, $J=6.5 \mathrm{~Hz}$ ), 7.00 (d, 1H, H-4, $J=8.4 \mathrm{~Hz}), 7.04-7.11(\mathrm{~m}, 2 \mathrm{H}, \mathrm{H}-2, \mathrm{H}-3)$, $7.20(\mathrm{~d}, 1 \mathrm{H}, \mathrm{H}-1, J=7.1 \mathrm{~Hz}) ;{ }^{19} \mathrm{~F}$ NMR $\left(376 \mathrm{MHz}, \mathrm{CDCl}_{3}\right): \delta=94.0(\mathrm{~d}$, $\mathrm{CF}_{3}, J=6.5 \mathrm{~Hz}$ ). Found: $\mathrm{C}, 54.80 ; \mathrm{H}, 4.49 ; \mathrm{N}, 8.51$. Calcd for $\mathrm{C}_{15} \mathrm{H}_{15} \mathrm{~F}_{3} \mathrm{~N}_{2} \mathrm{O}_{3}$ : C, 54.88; $\mathrm{H}, 4.61 ; \mathrm{N}, 8.53$.

4.4.2. $\left(6 S^{*}, 6 a S^{*}, 6 b S^{*}, 11 a R^{*}\right)-2-$ Bromo-6a-nitro-6-(trifluoromethyl)6,6a,6b,7,8,9,11,11a-octahydrochromeno[3,4-a]pyrrolizine (4b). This compound was prepared from 6-bromo-3-nitro-2-(trifluoromethyl)$2 \mathrm{H}$-chromene (1b), proline, and paraformaldehyde according to the described procedure for compounds 2 . Yield $15 \%$, mp $132-133{ }^{\circ} \mathrm{C}$, 
a white powder; IR (KBr): $\nu=1579,1550,1480,1372 \mathrm{~cm}^{-1} ;{ }^{1} \mathrm{H}$ NMR $\left(500 \mathrm{MHz}, \mathrm{CDCl}_{3}\right): \delta=1.53$ (ddt, $\left.1 \mathrm{H}, \mathrm{H}-7^{\prime}, J=13.2,8.6,8.1 \mathrm{~Hz}\right), 1.85(\mathrm{~m}$, $\left.1 \mathrm{H}, \mathrm{H}-8^{\prime}\right), 1.94$ (m, 1H, H-8"), 2.12 (dtd, 1H, H-7", J=13.2, 7.4, $4.1 \mathrm{~Hz}$ ), 2.70 (ddd, $1 \mathrm{H}, \mathrm{H}-9^{\prime}, J=10.0,8.5,6.6 \mathrm{~Hz}$ ), 3.09 (ddd, $1 \mathrm{H}, \mathrm{H}-9^{\prime \prime}, J=10.0$, 6.9, $3.8 \mathrm{~Hz}$ ), 3.28 (dd, $1 \mathrm{H}, \mathrm{H}-11^{\prime}, J=10.8,5.1 \mathrm{~Hz}$ ), 3.56 (dd, $1 \mathrm{H}, \mathrm{H}-11^{\prime \prime}$, $J=10.8,7.2 \mathrm{~Hz}$ ), 4.17 (t, 1H, H-6b, $J=7.5 \mathrm{~Hz}$ ), 4.26 (dd, 1H, H-11a, J=6.5, $5.5 \mathrm{~Hz}), 4.89$ (q, 1H, H-6, $\left.{ }^{3} J_{\mathrm{H}, \mathrm{F}}=6.4 \mathrm{~Hz}\right), 6.89(\mathrm{~d}, 1 \mathrm{H}, \mathrm{H}-4, J=8.4 \mathrm{~Hz})$, 7.30-7.33 (m, 2H, H-1, H-3); ${ }^{19} \mathrm{~F}$ NMR (376 MHz, $\left.\mathrm{CDCl}_{3}\right): \delta=93.9$ (d, $\mathrm{CF}_{3}, J=6.4 \mathrm{~Hz}$ ); ${ }^{13} \mathrm{C} \mathrm{NMR}\left(126 \mathrm{MHz}, \mathrm{CDCl}_{3}\right): \delta=26.1$ (C-8), 28.8 (C-7), 42.8 (C-11a), 55.0 (C-9), 61.1 (C-11), 70.7 (q, C-6b, $\left.{ }^{4} J_{C, F}=2.2 \mathrm{~Hz}\right), 74.1$ (q, $\left.\mathrm{C}-6,{ }^{2} J_{\mathrm{C}, \mathrm{F}}=32.0 \mathrm{~Hz}\right), 92.8(\mathrm{C}-6 \mathrm{a}), 115.9(\mathrm{C}-2), 118.9(\mathrm{C}-4), 122.9\left(\mathrm{q}, \mathrm{CF}_{3}\right.$, $\left.{ }^{1} J_{C, F}=285.6 \mathrm{~Hz}\right), 124.4$ (C-11b), 131.1 (C-1), 131.6 (C-3), 149.8 (C-4a). Found: $\mathrm{C}, 44.53 ; \mathrm{H}, 3.45 ; \mathrm{N}, 6.83$. Calcd for $\mathrm{C}_{15} \mathrm{H}_{14} \mathrm{BrF}_{3} \mathrm{~N}_{2} \mathrm{O}_{3}$ : C, 44.25; $\mathrm{H}, 3.47 ; \mathrm{N}, 6.88$.

4.4.3. (6S*,6aS*,6bS*,11aR*)-2-Bromo-6a-nitro-6-(trichloromethyl)6,6a,6b,7,8,9,11,11a-octahydrochromeno[3,4-a]pyrrolizine (4c). This compound was prepared from 6-bromo-3-nitro-2-(trichloromethyl)$2 \mathrm{H}$-chromene (1f), proline, and paraformaldehyde according to the described procedure for compounds 2 . Combine yield $11 \%, \mathrm{mp}$ $139-140^{\circ} \mathrm{C}$, a white powder; $\operatorname{IR}(\mathrm{KBr}): \nu=1548,1481,1441,1338 \mathrm{~cm}^{-1}$; ${ }^{1} \mathrm{H}$ NMR (400 MHz, $\left.\mathrm{CDCl}_{3}\right)$ : major isomer $4 \mathbf{c}(68 \%) \delta=1.5-3.1(\mathrm{~m}, 6 \mathrm{H}$, $3 \mathrm{CH}_{2}$ ), 3.28 (dd, $1 \mathrm{H}, \mathrm{H}-11^{\prime}, J=10.8,5.6 \mathrm{~Hz}$ ), 3.41 (dd, $1 \mathrm{H}, \mathrm{H}-11^{\prime \prime}, J=10.8$, $7.7 \mathrm{~Hz}), 4.39$ (t, 1H, H-6b, J=6.7 Hz), 4.59 (t, 1H, H-11a, J=7.6 Hz), 5.06 $(\mathrm{s}, 1 \mathrm{H}, \mathrm{H}-6), 6.93(\mathrm{~d}, 1 \mathrm{H}, \mathrm{H}-4, J=8.6 \mathrm{~Hz}), 7.30(\mathrm{~d}, 1 \mathrm{H}, \mathrm{H}-1, J=2.0 \mathrm{~Hz}), 7.32$ $(\mathrm{dd}, 1 \mathrm{H}, \mathrm{H}-3, J=8.6,2.0 \mathrm{~Hz})$; minor isomer $4 \mathbf{c}(32 \%): \delta=1.90-2.05(\mathrm{~m}$, $\left.4 \mathrm{H}, 2 \mathrm{CH}_{2}\right), 2.78\left(\mathrm{t}, 1 \mathrm{H}, 11^{\prime}, J=8.9 \mathrm{~Hz}\right), 2.86-3.08\left(2 \mathrm{H}, \mathrm{CH}_{2}\right), 3.70(\mathrm{t}, 1 \mathrm{H}$, $\left.\mathrm{H}-11^{\prime \prime}, J=8.9 \mathrm{~Hz}\right), 4.56(\mathrm{~s}, 1 \mathrm{H}, \mathrm{H}-6), 4.57(\mathrm{t}, 1 \mathrm{H}, \mathrm{H}-11 \mathrm{a}, J=7.6 \mathrm{~Hz}), 4.61$ (t, $1 \mathrm{H}, \mathrm{H}-6 \mathrm{~b}, J=6.5 \mathrm{~Hz}), 7.02(\mathrm{~d}, 1 \mathrm{H}, \mathrm{H}-4, J=8.6 \mathrm{~Hz}), 7.19-7.22(\mathrm{~m}, 1 \mathrm{H}, \mathrm{H}-1)$, 7.34-7.39 (m, 1H, H-3). Found: C, 39.38; H, 3.13; N, 6.09. Calcd for $\mathrm{C}_{15} \mathrm{H}_{14} \mathrm{BrCl}_{3} \mathrm{~N}_{2} \mathrm{O}_{3}$ : C, 39.46; $\mathrm{H}, 3.09 ; \mathrm{N}, 6.14$.

4.4.4. (6R*,6aS*,6bS*,11aR*)-6a-Nitro-6-phenyl-6,6a,6b,7,8,9,11,11aoctahydrochromeno[3,4-a]pyrrolizine (4d). This compound was prepared from 3-nitro-2-phenyl-2H-chromene (1i), proline, and paraformaldehyde according to the described procedure ${ }^{6 e}$ as a base and purified by recrystallization from hexane/ether. Yield 34\%, mp 161-163 ${ }^{\circ} \mathrm{C}$, yellow crystals; IR (KBr): $\nu=1611,1587,1535,1489$, $1457,1374 \mathrm{~cm}^{-1}$; ${ }^{1} \mathrm{H}$ NMR (500 MHz, $\mathrm{CDCl}_{3}$ ): $\delta=1.40$ (dtd, $1 \mathrm{H}, \mathrm{H}-7^{\prime}$, $J=12.8,9.1,8.5 \mathrm{~Hz}$ ), 1.74 (ddt, $1 \mathrm{H}, \mathrm{H}-8^{\prime}, J=12.5,9.6,7.5 \mathrm{~Hz}$ ), $1.85-1.97$ (m, 2H, H-7", H-8"), 2.83 (ddd, 1H, H-9', J=10.6, 8.1, $6.5 \mathrm{~Hz}$ ), 3.21 (ddd, 1H, H-9", $J=10.6,7.0,4.2 \mathrm{~Hz}$ ), 3.37 (dd, 1H, H-11', $J=11.7$, $9.3 \mathrm{~Hz}$ ), 3.43 (dd, 1H, H-11", $J=11.7,9.3 \mathrm{~Hz}$ ), 3.84 (dd, 1H, H-6b, $J=9.4,6.8 \mathrm{~Hz}$ ), 4.14 (t, 1H, H-11a, $J=9.3 \mathrm{~Hz}$ ), 5.28 (s, 1H, H-6), 6.96 (dd, $1 \mathrm{H}, \mathrm{H}-4, J=8.1,1.1 \mathrm{~Hz}$ ), 7.02 (td, $1 \mathrm{H}, \mathrm{H}-2, J=7.5,1.1 \mathrm{~Hz}$ ), 7.15-7.20 $(\mathrm{m}, 2 \mathrm{H}, \mathrm{H}-1, \mathrm{H}-3), 7.40-7.43(\mathrm{~m}, 5 \mathrm{H}, \mathrm{Ph}) ;{ }^{13} \mathrm{C}$ NMR $(126 \mathrm{MHz}$, $\mathrm{CDCl}_{3}$ ): $\delta=25.0(\mathrm{C}-8), 30.0$ (C-7), 40.4 (C-11a), 57.0 (C-9), 61.7 (C-11), 68.3 (C-6b), 78.6 (C-6), 96.1 (C-6a), 117.3 (C-4), 122.17 (C-11b), 122.21 (C-2), 127.94 (C-3), 127.96 (Co), 128.1 (Cm), 128.8 (C-1), 129.2 (Cp), 134.5 (Ci), 153.7 (C-4a). Found: C, 71.26; H, 6.08; N, 8.29. Calcd for $\mathrm{C}_{20} \mathrm{H}_{20} \mathrm{~N}_{2} \mathrm{O}_{3}$ : C, 71.41; H, 5.99; N, 8.33.

\subsection{Compounds 5a,b}

4.5.1. (3aR*,9bR*)-2-Methyl-1,2,3,3a,4,9b-hexahydrochromeno[3,4-c] pyrrole-3a-carbonitrile (5a). This compound was prepared as a yellow oil from 3-cyano- $2 \mathrm{H}$-chromene $(\mathbf{1 j})$, sarcosine, and paraformaldehyde according to the described procedure. ${ }^{6 e}$ Yield $76 \%$, $\mathrm{mp}>210{ }^{\circ} \mathrm{C}$ (subl.), a white powder (for hydrochloride); IR ( $\mathrm{KBr}$ ): $\nu=2638,2568,2440,2245,1607,1580,1490,1470,1456,1412$, $1344 \mathrm{~cm}^{-1} ;{ }^{1} \mathrm{H}$ NMR (400 MHz, $\mathrm{CDCl}_{3}$ ): $\delta=2.36$ (s, 3H, Me), 2.50 (dd, $\left.1 \mathrm{H}, \mathrm{H}-1^{\prime}, J=9.2,7.5 \mathrm{~Hz}\right), 2.66\left(\mathrm{~d}, 1 \mathrm{H}, \mathrm{H}-3^{\prime}, J=10.0 \mathrm{~Hz}\right), 3.30$ (d, $1 \mathrm{H}, \mathrm{H}-$ $\left.3^{\prime \prime}, J=10.0 \mathrm{~Hz}\right), 3.33\left(\mathrm{t}, 1 \mathrm{H}, \mathrm{H}-1^{\prime \prime}, J=8.7 \mathrm{~Hz}\right), 3.72(\mathrm{t}, 1 \mathrm{H}, \mathrm{H}-9 \mathrm{~b}$, $J=7.7 \mathrm{~Hz}), 3.91$ (d, $\left.1 \mathrm{H}, \mathrm{H}-4^{\prime}, J=11.2 \mathrm{~Hz}\right), 4.23$ (d, $\left.1 \mathrm{H}, \mathrm{H}-4^{\prime \prime}, J=11.2 \mathrm{~Hz}\right)$, $6.95(\mathrm{~d}, 1 \mathrm{H}, \mathrm{H}-6, J=8.4 \mathrm{~Hz}), 6.99(\mathrm{t}, 1 \mathrm{H}, \mathrm{H}-8, J=7.4 \mathrm{~Hz}), 7.10(\mathrm{~d}, 1 \mathrm{H}, \mathrm{H}-$ 9, $J=7.3 \mathrm{~Hz}$ ), 7.17 (t, 1H, H-7, J=7.7 Hz); ${ }^{13} \mathrm{C}$ NMR (126 MHz, DMSO- $\left.d_{6}\right): \delta=37.5,38.8,41.3,57.7,58.1,65.5,117.3,117.5,119.8,122.2,129.1$, 130.1, 153.0. Found: C, 62.36; $\mathrm{H}, 6.23 ; \mathrm{N}, 10.88$. Calcd for $\mathrm{C}_{13} \mathrm{H}_{14} \mathrm{~N}_{2} \mathrm{O} \cdot \mathrm{HCl}: \mathrm{C}, 62.28 ; \mathrm{H} 6.03 ; \mathrm{N}, 11.17$.

4.5.2. ( $\left.3 a R^{*}, 9 b R^{*}\right)$-Methyl 2-methyl-1,2,3,3a,4,9b-hexahydrochrome no[3,4-c]pyrrole-3a-carboxylate (5b). This compound was prepared as a yellow oil from 3-methoxycarbonyl- $2 \mathrm{H}$-chromene (1k), sarcosine, and paraformaldehyde according to the described procedure ${ }^{6 e}$ Yield $83 \%$, mp 202-204 ${ }^{\circ} \mathrm{C}$, a white powder (for hydrochloride); IR (KBr): $\nu=2680,2588,2495,2364,1723,1612$, 1586, 1492, 1458, 1439, 1358, $1345 \mathrm{~cm}^{-1} ;{ }^{1} \mathrm{H}$ NMR $(400 \mathrm{MHz}$, $\left.\mathrm{CDCl}_{3}\right): \delta=2.34(\mathrm{~s}, 3 \mathrm{H}, \mathrm{Me}), 2.45-2.51\left(\mathrm{~m}, 2 \mathrm{H}, \mathrm{H}-3^{\prime}, \mathrm{H}-1^{\prime}\right), 3.21(\mathrm{~d}$, $1 \mathrm{H}, \mathrm{H}-3^{\prime \prime}, J=10.0 \mathrm{~Hz}$ ), 3.34 (t, $1 \mathrm{H}, \mathrm{H}-1^{\prime \prime}, J=8.5 \mathrm{~Hz}$ ), 3.74 (s, 3H, MeO), 3.85 (d, $\left.1 \mathrm{H}, \mathrm{H}-4^{\prime}, J=11.0 \mathrm{~Hz}\right), 3.92(\mathrm{t}, 1 \mathrm{H}, \mathrm{H}-9 \mathrm{~b}, J=8.0 \mathrm{~Hz}), 4.38(\mathrm{~d}, 1 \mathrm{H}$, $\left.\mathrm{H}-4^{\prime \prime}, J=11.0 \mathrm{~Hz}\right), 6.89$ (d, 1H, H-6, $\left.J=8.1 \mathrm{~Hz}\right), 6.94(\mathrm{t}, 1 \mathrm{H}, \mathrm{H}-8$, $J=7.5 \mathrm{~Hz}$ ), 7.08-7.16 (m, 2H, H-7, H-9); ${ }^{13} \mathrm{C}$ NMR (126 MHz, DMSO$\left.d_{6}\right): \delta=38.1,49.2,52.9,57.7,58.9,67.3,117.0,120.3,121.8,128.2$, 129.7, 153.6, $171.5(\mathrm{C}=\mathrm{O})$ (one $\mathrm{C}$ is masked). Found: $\mathrm{C}, 59.13 ; \mathrm{H}$, 6.49; $\mathrm{N}, 4.93$. Calcd for $\mathrm{C}_{14} \mathrm{H}_{17} \mathrm{NO}_{3} \cdot \mathrm{HCl}$ : C, 59.26; $\mathrm{H}, 6.39 ; \mathrm{N}, 4.84$.

\subsection{X-ray diffraction study of compound $2 b$}

Intensities of reflections were measured on an automatic fourcircle Xcalibur 3 diffractometer (Oxford Diffraction, Abingdon, UK; graphite monochromated Mo K $\alpha$ radiation, CCD detector, $\omega$ scanning). The structure was solved by a direct method using the SHELX97 package. ${ }^{13}$ The hydrogen atoms were located geometrically using a riding model. Nonhydrogen atoms were refined by a full-matrix least-squares method against $F^{2}$ within the anisotropic approximation. Final atomic coordinates, geometrical parameters, and crystallographic data have been deposited with the Cambridge Crystallographic Data Centre (12 Union Road, Cambridge CB2 1EZ, UK; fax: +44 1223 336033; e-mail: deposit@ccdc.cam.ac.uk). The CCDC deposition number for compound 2b is 925671. These data can be obtained free of charge from the Cambridge Crystallographic Data Centre via http://www.ccdc.cam.ac.uk/products/csd/request/.

Crystal data for 2b at 295(2) K: $\mathrm{C}_{13} \mathrm{H}_{12} \mathrm{BrF}_{3} \mathrm{~N}_{2} \mathrm{O}_{3}, M=381.15$, $a=7.3625(14) \AA, b=7.6363(12) \AA, c=13.950(3) \AA, \alpha=74.399(15)^{\circ}$, $\beta=78.411(15)^{\circ}, \quad \gamma=75.659(15)^{\circ}, V=724.2(2) \AA^{3}$, triclinic crystals space group $P \overline{1}, Z=2, D_{\mathrm{c}}=1.748 \mathrm{~g} \mathrm{~cm}^{-3}, \mu=2.885 \mathrm{~mm}^{-1}, F(000)=$ 380,7403 reflections measured up to $2 \theta_{\max }=56.6^{\circ}, 3492$ unique $\left(R_{\text {int }}=0.0309\right)$, which were used in all calculations. Refinement was converged at $w R_{2}=0.0557$ (all data), $R_{1}=0.0324$ (1551 reflections with $I>2 \sigma(I)), \mathrm{GoF}=1.001$.

\section{Acknowledgements}

This work was supported financially by the Russian Foundation for Basic Research (Grants 11-03-00126 and 12-03-31036).

\section{References and notes}

1. Padwa, A.; Pearson, W. H. Synthetic Applications of 1,3-Dipolar Cycloaddition Chemistry Toward Heterocycles and Natural Products; Wiley: New York, NY 2002; Vol. 59, p169.

2. (a) Tsuge, O.; Kanemasa, S. Adv. Heterocycl. Chem. 1989, 45, 231; (b) Pandey, G.; Banerjee, P.; Gadre, S. R. Chem. Rev. 2006, 106, 4484; (c) Nájera, C.; Sansano, J. M. Curr. Org. Chem. 2003, 7, 1105; (d) Coldham, I.; Hufton, R. Chem. Rev. 2005, 105, 2765.

3. (a) Horton, D. A.; Bourne, G. T.; Smythe, M. L. Chem. Rev. 2003, 103, 893; (b) Razdan, R. K.; Howes, J. F. Med. Res. Rev. 1983, 3, 119.

4. (a) Dubuffet, T.; Muller, O.; Simonet, S. S.; Descombes, J.-J.; Laubie, M.; Verbeuren, T. J.; Lavielle, G. Bioorg. Med. Chem. Lett. 1996, 6, 349; (b) Dubuffet, T; Newman-Tancredi, A.; Cussac, D. Audinot, V.; Loutz, A. Millan, M. J.; Lavielle, G. Bioorg. Med. Chem. Lett. 1999, 9, 2059; (c) Lavielle, G.; Dubuffet, T.; Millan, M.; Newman-Tancredi, A. U.S. Patent 5,663,191, 1997; (d) Meyer, M. D.; Altenbach, R. J.; Basha, F. Z.; Carroll, W. A.; Drizin, I.; Kerwin, J. F.; Wendt, M. D.; Haight, A. R.; Zhang, W. U.S. Patent 6,046,207, 2000; (e) Kiyoi, T.; Reid, M.; Francis, S.; Davies, K.; Laats, S.; McArthur, D.; 
Easson, A.-M.; Kiyoi, Y.; Tarver, G.; Caulfield, W.; Gibson, K.; Wishart, G.; Morrison, A. J.; Adam, J. M.; Ray, P. Tetrahedron Lett. 2011, 52, 3413; (f) Ghandi, M.; Taheri, A.; Abbasi, A. Tetrahedron 2010, 66, 6744; (g) Pars, H. G.; Granchelli, F. E.; Razdan, R. K.; Keller, J. K.; Teiger, D. G.; Rosenberg, F. J.; Harris, L. S. J. Med. Chem. 1976, 19, 445.

5. Haight, A. R.; Bailey, A. E.; Baker, W. S.; Cain, M. H.; Copp, R. R.; DeMattei, J. A.; Ford, K. L.; Henry, R. F.; Hsu, M. C.; Keyes, R. F.; King, S. A.; McLaughlin, M. A.; Melcher, L. M.; Nadler, W. R.; Oliver, P. A.; Parekh, S. I.; Patel, H. H.; Seif, L. S.; Staeger, M. A.; Wayne, G. S.; Wittenberger, S. J.; Zhang, W. Org. Process Res. Dev. 2004, 8, 897.

6. (a) Grigg, R.; Vipond, D. Tetrahedron 1989, 45, 7587; (b) Grigg, R.; Idle, J.; McMeekin, P.; Surendrakumar, S.; Vipond, D. J. Chem. Soc., Perkin Trans. 1 1988, 2703; (c) Moshkin, V. S.; Sosnovskikh, V. Y.; Slepukhin, P. A.; Röschenthaler, G.V. Mendeleev Commun. 2012, 22, 29; (d) Moshkin, V. S.; Sosnovskikh, V. Y.; Röschenthaler, G.-V. Tetrahedron Lett. 2012, 53, 3568; (e) Moshkin, V. S.; Sosnovskikh, V. Y.; Röschenthaler, G.-V. Tetrahedron 2013, 69, 5884.

7. (a) Nyerges, M.; Virányi, A.; Marth, G.; Dancsó, A.; Blaskó, G.; Tőke, L. Synlett 2004, 2761; (b) Virányi, A.; Marth, G.; Dancsó, A.; Blaskó, G.; Tőke, L.; Nyerges, M. Tetrahedron 2006, 62, 8720.

8. Nyerges, M.; Balázs, L.; Kádas, I.; Bitter, I.; Kövesdi, I.; Tőke, L. Tetrahedron 1995 $51,6783$.
9. (a) Organofluorine Compounds in Medicinal Chemistry and Biomedical Applications; Filler, R., Kobayashi, Y., Yagupolskii, L. M., Eds.; Elsevier: Amsterdam, The Netherlands, 1993; (b) Hiyama, T. Organofluorine Compounds: Chemistry and Application; Springer: Berlin, Germany, 2000.

10. Korotaev, V. Y.; Kutyashev, I. B.; Sosnovskikh, V. Y. Heteroat. Chem. 2005 $16,492$.

11. (a) Korotaev, V. Y.; Sosnovskikh, V. Y.; Kutyashev, I. B.; Kodess, M. I. Lett. Org Chem. 2005, 2, 616; (b) Korotaev, V. Y.; Sosnovskikh, V. Y.; Kutyashev, I. B. Kodess, M. I. Izv. Akad. Nauk, Ser. Khim. 2006, 309; [ Russ. Chem. Bull., Int. Ed. 2006, 55, 317 ]; (c) Korotaev, V. Y.; Sosnovskikh, V. Y.; Kutyashev, I. B.; Kodess, M. I. Izv. Akad. Nauk, Ser. Khim. 2006, 1945; [ Russ. Chem. Bull., Int. Ed. 2006, 55, 2020 ]; (d) Korotaev, V. Y.; Sosnovskikh, V. Y.; Kutyashev, I. B. Izv. Akad. Nauk, Ser. Khim. 2007, 1985; [ Russ. Chem. Bull., Int. Ed. 2007, 56, 2054 ]; (e) Korotaev, V. Y.; Kutyashev, I. B.; Sosnovskikh, V. Y.; Kodess, M. I. Mendeleev Commun. 2007, 17, 52; (f) Korotaev, V. Y.; Sosnovskikh, V. Y.; Barkov, A. Y.; Slepukhin, P. A.; Ezhikova, M. A.; Kodess, M. I.; Shklyaev, Y. V. Tetrahedron 2011, 67, 8685.

12. Korotaev, V. Y.; Sosnovskikh, V. Y.; Barabanov, M. A.; Yasnova, E. S.; Ezhikova, M. A.; Kodess, M. I.; Slepukhin, P. A. Tetrahedron 2010, 66, 1404

13. Sheldrick, G. M. Acta Crystallogr., Sect. A 2008, 64, 112 